\title{
Heat Transfer Performance of a Gasketed Plate Heat Exchanger using Nanofluid and Subject to Vibration as a Combined Augmentation Techniques
}

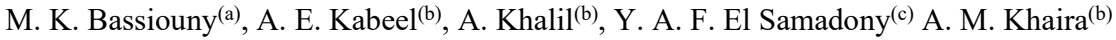 \\ (a) Mechanical Power Eng. Dept. Faculty of Engineering, Menoufia University, Egypt. \\ (b) Mechanical Power Eng. Dept. Faculty of Engineering, Tanta University, Egypt.

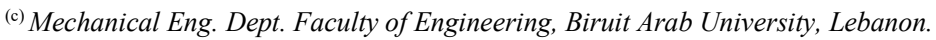

\begin{abstract}
In the present study, an experimental investigation for heat transfer performance of $\gamma \mathrm{Al2O}$-water nanofluid through a gasketed plate heat exchanger (GPHE) subjected to a low amplitude mechanical vibration was studied. $\gamma$ Al2O3-water nanofluid with four different volume fractions have been prepared and used as the hot working fluid. Corrugated plate heat exchanger (PHE) with six stainless steel plates; creating three cold municipal water channels and two hot nanofluid channels in counter flow pattern. GPHE is subjected to vibration by means of a grinding motor. Vibration frequencies is varied from 13.33 to $46.67 \mathrm{cps}$ and vibrational dimensionless amplitude (A/De) is varied from $9.14 \times 10^{-3}$ to $52.66 \times 10^{-3}$. The experiments have been conducted in the Reynolds number ranging from 730 to 3400 . The results revealed that as vibrational dimensionless amplitude (A/De) and nanofluid volume fractions increases the net heat transfer coefficient enhancement ratio increases. The net heat transfer coefficient increases as mechanical vibration amplitude decreases. The maximum net heat transfer coefficient enhancement ratio is $64 \%$, which is achieved at $0.51 \%$ volume fraction and oscillation Reynolds number of 211.34, which was occur just after the resonance condition. Moreover, a new empirical correlation is proposed to predict the GPHE Nusselt number Finally, pressure drop is measured and found that it is increased as nanofluid volume fraction increased.
\end{abstract}

Keywords-Nanofluid, mechanical vibration, heat transfer augmentation, gasketed plate heat exchanger.

\section{INTRODUCTION}

$\mathbf{P}$ LATE Heat Exchangers (PHEs) are broadly used in numerous engineering applications. Currently, the rapid technology development has focused on the possible techniques of heat transfer enhancement in numerous engineering systems. These techniques are classified to passive or active. Some such techniques tend to use of fluid with superior transport properties (as a kind of passive enhancement techniques) and vibrating the heat transfer surface (as a kind of active enhancement techniques. Choi and Estman [1] were the earliest to employ the nanometersized particles in traditional fluids and showed considerable increase in the nanofluid thermal conductivity. Therefore, Nanofluid have received considerable scientific interest during the last decade [2-6]. The suitable physical mechanism of heat transfer augmentation has not been confirmed till now. It must be noted that the increase of thermal conductivity might be offset by the viscosity increase and hence pressure drop. Surface vibrations is one of the active techniques, which increase turbulence intensity in boundary layer and hence fluid mixing, which enhance the heat transfer performance.
Heat exchanger heat transfer performance and pressure drop were studied experimentally and theoretically with and without Nanofluid [7]. Pantzali et al. [8] studied experimentally and numerically the effect of use $\mathrm{CuO} /$ water with $4 \%$ vol. fraction nanofluid on the performance of a miniature PHE with modulated surface. They found that the heat transfer rate was enhanced and nanofluid volumetric flow rate was lower than that of water, causing lower pressure drop and pumping power. Pantzali et al. [9] They also concluded that the high price and the potential instability of suspension were two major disadvantages of a nanofluid. Maré et al. [10] compared experimentally the thermal performances of two types of commercial nanofluids. Their results showed considerable improvement in laminar mode of the convective heat transfer coefficient by about $42 \%$ and $50 \%$ for $\mathrm{Al} 2 \mathrm{O} 3 /$ water and CNT/water nanofluids respectively for the same Reynolds number. Zamzamian et al. [11] found that a considerable enhancement in the convective heat transfer coefficient ranging from $2 \%$ to $50 \%$ in a double pipe and PHE when nanofluid was used. Kwon et al. [12]. carried out an experimental investigation on heat transfer characteristics and pressure drop of the $\mathrm{ZnO}$ /water and $\mathrm{Al} 2 \mathrm{O} 3$ /water nanofluids in a PHE. They concluded that the OHTC increased by $30 \%$ at $6 \%$ vol. concentration of $\mathrm{Al} 2 \mathrm{O} 3$ nanofluid. However, they concluded that the application of nanofluids in PHE remains inconclusive. Pandey and Nema [13] investigated experimentally the effect of $\mathrm{Al} 2 \mathrm{O} 3$ nanofluid with 2, 3 and 4 vol.\% on heat transfer and frictional losses in a counter flow corrugated PHE. It was observed that the heat transfer characteristics are improved with the increase in Reynoldsand-Peclet- number and with decrease in nanofluid concentration. For a given heat load, the required pumping power increased with increase in nanofluid concentration. They suggested avoiding more than 2 vol.\% fraction of this nanofluid due to economic consideration due to nanoparticle price and pumping operating cost. Tiwari et al. [14] investigated experimentally effect of using various nanofluids for wide range of concentrations on the performance of a PHE. They concluded that various nanofluids have different optimum volume concentration in which the heat transfer characteristics showed the maximum enhancement. Ray et al. [15] studied the influence of three types of nanofluids. They observed that using nanofluids was not only reduced pumping power and size of minichannel PHE but also achieved the same amount of heat transfer. Kumar et al. [16] introduced an experimental study about the effect of variable spacing on performance of PHE using nanofluids. Various nanoparticles are used in their study. They concluded that MWCNT/water nanofluid at $0.75 \mathrm{vol} \%$. with a plate spacing of $5.0 \mathrm{~mm}$, achieved the maximum heat transfer coefficient, which is $53 \%$ 
higher compared to water. The experiments also recorded a nominal rise in pressure drop at $0.75 \mathrm{vol} \%$. Kabeel et al. [17] executed experiments to study chevron-type corrugated PHE heat transfer characteristics and pressure drop when using different volumetric concentrations of Al2O3/water nanofluid. The heat transfer coefficient was increased by $13 \%$ at $4 \%$ vol. nanofluid concentration. The maximum increase in pressure drop was recorded $45 \%$ above the base fluid value at $4 \%$ vol. of nanofluid, while the pumping power was recorded $90 \%$ higher due to the effect of increase in both the pressure drop and the volumetric flow rate. Abed et al. [18] studied numerically heat transfer behavior of nanofluids in trapezoidal PHE. This study evaluated the effects of four different types of nanoparticles with different volume fractions. Their results demonstrated that $\mathrm{SiO} 2$ had the highest Nusselt number among the different nanofluids.

Vibration can be observed as a powerful means for heat transfer augmentation. However, most of vibration researches were executed on a cylindrical or a spherical surface [19]. The influence of vibration on heat transfer for different geometries was conducted [20-24]. Prasad and Ramanathan [20] studied experimentally the effect of longitudinal oscillations on the free convective heat transfer coefficient for a hot vertical plate. They showed that the vibration caused a significant increase in steady rate heat transfer especially at low Rayliegh number. They found also that the maximum increase in heat transfer rate was 33\%. Eid and Gomaa [21] investigated the effect of vibration on heat transfer rate for thin planar fins. They found that the augmentation in rates of heat transfer reached $85 \%$ or more via normal vibration. Sarhan [22] conducted the investigation of the influence of vibration on the free convection performance for longitudinally finned plate. They reported a good enhancement in the heat transfer rate when vibrations were applied. Klaczak [23] carried out investigations concerns with the effect of vibrations on a horizontal steam-water heat exchanger. The vibration was in the same direction of the steam-water flow. It was clarified that the vibrations with high acceleration improve heat transfer in general. Chatter and Sandeep [24] investigated the effect of vibration on a horizontal rectangular heat exchanger with five triangular baffles inclined at fixed angle of $20^{\circ}$ along the channel. Their results found that the overall heat transfer coefficient showed the maximum increase of $70 \%$. The maximum effectiveness of $60.37 \%$ was found on vibration level of 6.5. Bassiouny et al. [25] experimentally investigated the effect of low amplitude mechanical vibration applied on a vertical counter flow GPHE on the heat transfer characteristics and heat exchanger effectiveness. The examined GPHE is made of stainless-steel plates with $30^{\circ}$ chevron angle. Six plates were installed; providing two hot channels and three cold channels in counter flow arrangement. They found that the heat transfer performance of the GPHE is enhanced when vibration is applied. The maximum enhancement percentage of the GPHE heat transfer coefficient, overall heat transfer coefficient and effectiveness due to vibration are $43 \%, 31 \%$ and $18 \%$, respectively. These maximums are occurred at oscillation Reynolds number of 211.34 and $\mathrm{A} / \mathrm{De}=52.66 \times 10^{-3}$ which after the resonance condition.

To the authors knowledge, the combined effects of nanofluids and vibration on natural or forced convection heat transfer of a GPHE is not studied experimentally or theoretically. The present work will do this novel study. To see the combined effect of adding Nanofluid to mechanical vibration Bassiouny et al. [25] experimental model will be used. The effects of nanofluid and mechanical vibrations on a gasketed plate heat exchanger (GPHE) performance will be studied. Moreover, the effect of changing the working parameters of the nanofluid and vibration on the GPHE performance will be obtained. Finally, the net heat transfer characteristics of nanofluids with mechanical vibration imposed on the PHE surfaces.

\section{EXPERIMENTAL SETUP}

Experimental set up will cover nanofluid preparation, and the used test rig.

\section{A. Preparation of nanofluid}

Distilled water was used as the base fluid. A commercially available Aluminum oxide nanoparticles (A12O3), $30 \mathrm{~nm}$ in diameter, was used. Full specifications of tested nano-fluid are summarized in Table (1). To prepare the nanofluid, a twostep was used. Firstly, adequate surfactant (SDBS) was dissolved in to the distilled water, to ensure better stability and proper dispersion without affecting nano fluids thermophysical properties (the surfactant concentration in the experiments is $0.01 \%$ ), the nanoparticles were added gradually in to the base fluid. An appropriate amount of nanoparticles is weighed out via analytical balance. The nanoparticles then add to $1 \mathrm{~L}$ of distilled water contained in a $1.2 \mathrm{~L}$ beaker. The mixture is stirred by magnetic stirrer at $1500 \mathrm{rpm}$ for 30 minutes. A combination of a bath-type and probe-type ultrasonicator for dispersion of the particles. First, the ultrasonic bath was used for 1 hour and then let to sit for 10 minutes to dissipate any generated heat. This process is repeated four times with manual stirring of the solution before sonication is started again. Then, to ensure maximum possible de-agglomeration, a probe type ultrasonic homogenizer was used to disperse the $\mathrm{Al} 2 \mathrm{O} 3$ nanoparticles in the base fluid. The solution is sonicated at $80 \%$ amplitude for one hour and then let to sit for 10 minutes to dissipate any generated heat. This process is repeated four times with manual stirring of the solution before sonication is started again. Also, to prevent possible sedimentation, for each nanofluid weight concentration test a new nanofluid was prepared. The nanofluids with different weight concentrations, were prepared and tested in the hot loop. Volume fraction $\emptyset_{V}$ of the nanofluid can be obtained from its weight (mass) fraction $\emptyset_{m}$ as follow [26]:

$$
\emptyset_{V}=\frac{1}{\left(\frac{1-\emptyset_{m}}{\emptyset_{m}}\right) \frac{\rho_{p}}{\rho_{f}}+1}
$$

The volume fractions of the four tested nanofluids are $0.125 \%$ vol., $0.25 \%$ vol., $0.378 \%$ vol., and $0.51 \%$ vol., respectively.

\section{B. Experimental test rig}

In this work, an Alfa Laval gasket plate heat exchanger, GPHE, with commercial chevron plates is used. As illustrated from Fig. 1 the plate is made of stainless steel with chevron angle of $30^{\circ}$. The plates have surface enlargement factor of 1.17. The used gasket plate heat exchanger has six plates, providing two hot channels and three cold channels in counter flow arrangement. Fig. 2 represents a Schematic diagram of the experimental work test rig with instrumentation. A 100 
liters insulated tank are used for suppling hot water through a pump. Six independent immersion heaters, $3 \mathrm{~kW}$ in capacities, are used to heat the water inside the tank. On/off switch and a digital thermostat are used to make a control on the hot temperature to be within $\pm 1{ }^{\circ} \mathrm{C}$ deviation. For cold side, the municipal water is used. The amount water flow rate (hot or cold) is adjusted by two manually controlled valves.

TABLE-1

SPECIFICATIONS OF TESTED NANO-FLUID

\begin{tabular}{|c|c|}
\hline Parameters & Value / Type \\
\hline \multicolumn{2}{|c|}{ Nano particle material } \\
\hline Nano particle material & $\mathrm{Al}_{2} \mathrm{O}_{3}$ \\
\hline $\begin{array}{l}\text { Product model (according to data sheet } \\
\text { form supplier) }\end{array}$ & AL-01 \\
\hline Purity of nano particle material & $99.9 \%$ \\
\hline Appearance & White \\
\hline Nano particle size (diameter), $d_{n p}(m)$ & $30 \times 10^{-9}$ \\
\hline Specific surface area, $\left(\mathrm{m}^{2} / \mathrm{g}\right)$ & 160 \\
\hline Volume density, $\left(\mathrm{g} / \mathrm{cm}^{3}\right)$ & 0.916 \\
\hline Density, $\rho_{n p}\left(\mathrm{~kg} / \mathrm{m}^{3}\right)$ & 3910 \\
\hline Specific heat, $C_{p, n p}(\mathrm{~J} / \mathrm{kg} . K)$ & 880 \\
\hline Thermal conductivity, $k_{n p}(\mathrm{~W} / \mathrm{m} . \mathrm{K})$ & 36 \\
\hline Crystal Form & y (Gamma) \\
\hline Characteristics & Insoluble \\
\hline Particle shape & sphere \\
\hline \multicolumn{2}{|c|}{ Nano-fluid } \\
\hline Base fluid & Distilled water \\
\hline $\begin{array}{l}\text { Nano particle mass fraction, } \emptyset_{m} \\
\text { (dimensionless) }\end{array}$ & $\begin{array}{l}0.5 \% \text { wt., } 1.0 \% \text { wt., } 1.5 \% \text { wt., } \\
\text { and } 2.0 \% \text { wt. }\end{array}$ \\
\hline $\begin{array}{l}\text { Nano particle volume fraction, } \emptyset_{V} \\
\text { (dimensionless) }\end{array}$ & $\begin{array}{l}0.125 \% \text { vol., } 0.25 \% \text { vol., } 0.378 \% \\
\text { vol., and } 0.51 \% \text { vol. }\end{array}$ \\
\hline flow rate of nano fluid, $m_{n f}\left(\mathrm{~m}^{3} / \mathrm{s}\right)$ & $6,8,10,12,15,18$ and $21 \mathrm{l} / \mathrm{min}$ \\
\hline Dispersion condition & Agitation \\
\hline Base fluid $\mathrm{pH}$ & $5.83-6.23$ \\
\hline Dispersant agent & SDBS 1.0 wt. $\%$ \\
\hline
\end{tabular}

A $370 \mathrm{~W}$ with $2850 \mathrm{rpm}$ pump was utilized to circulate the hot water. Rotameters with an accuracy of \pm 0.04 were used to measure the hot and cold water volume flow rates. Accordingly, the water mass flow rates were calculated at mean temperature. To minimize heat loss from the heat exchanger, hot water tank and piping, are well insulated by polyurethane foam and aluminum foil.

Vibrations are generated by means of a grinding motor. The grinding wheel spindle is mounted on a sub-assembly, which is attached to the rigid platform by means of flexures which inhibit movement of the sub-assembly in all other directions. The grinding machine rigid platform is attached to the GPHE's platform by means of screws and is located in the left bottom side of the tested GPHE as shown in Fig. 3. The grinding motor is $350 \mathrm{~W}$ and operates at a wide range of speeds up to a maximum of 2950 RPM. A one horsepower, 208/240 input voltage variable speed AC inverter, is used to regulate the motor rotational speed and hence vibration intensity.

Temperature measurements are obtained by using high accuracy $( \pm 0.1 \mathrm{~K})$ J-type thermocouples, located at GPHE inlets and outlets. The temperatures are monitoring and recording by connecting the thermocouples to 8 channels USB Data Acquisition from Omega to PC. The GPHE pressure drop in the hot and cold side are measured by using four pressure transducers, whose accuracy $0.2 \%$. The pressure and temperature are recorded by using TRACER software. All measuring instruments are calibrated.
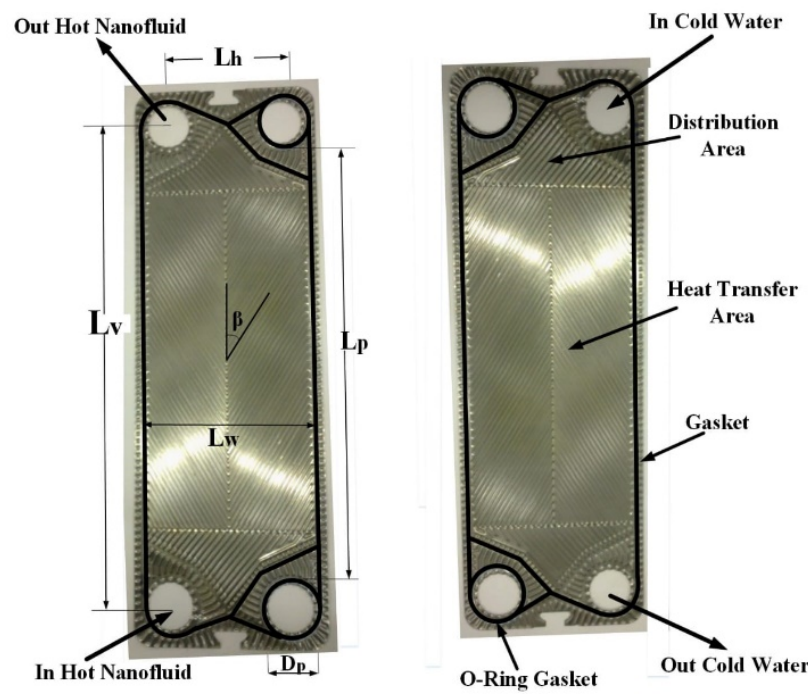

Fig. 1. Gasketed plate heat exchanger basic geometrical and flow characteristics.

Different vibrations intensities are used to find their influence on heat transfer performance. Two-channel one Prod MVP-2C accelerometer is used to detect vibration dynamic response and vibration excitation displacement at three positions on the tested GPHE. One Prod MVP-2C includes all tools required for vibration data such as, data collector, analyzer, recorder, balancer and order analysis.

To start the system, the two circulating pumps are switched on. The used amount of water heaters is determined according to the required or designed temperature of hot water. The hot water inlet temperature, set using a thermostat, is approximately constant at $65{ }^{\circ} \mathrm{C}$ during all experiments. The hot and cold-water flow rates were adjusted to desired settings by manually controlled needle and gate valves.

\section{Experimental data analysis}

The thermo-physical properties of $\mathrm{Al}_{2} \mathrm{O}_{3}$ /water nanofluid can be obtained by using classical formulas derived for a twophase mixture as follows: Pak and Cho, [27]

$$
\rho_{n f}=\left(1-\emptyset_{V}\right) \rho_{b f}+\emptyset_{V} \cdot \rho_{n p}
$$

where $\rho_{b f}$ is the density of base fluid and $\rho_{n p}$ is the density of nanoparticles. The specific heat capacity of nanofluid $C_{p_{n f}}$ is obtained by Xuan and Roetzel, [28], as follows:

$$
\left(\rho . C_{p}\right)_{n f}=\left(1-\emptyset_{V}\right)\left(\rho . C_{p}\right)_{b f}+\emptyset_{V}\left(\rho . C_{p}\right)_{n p}
$$
where $C_{p_{b f}}$ and $C_{p_{n p}}$ are the specific heat capacity of base fluid and nanoparticles respectively.

Thermal conductivity may be obtained as follows: Patel et al. [29]

$$
\frac{K_{e f f}}{K_{f}}=1+0.135 \emptyset_{V}^{0.467}\left(\frac{K_{p}}{K_{f}}\right)^{0.273}\left(\frac{T}{20}\right)^{0.547}\left(\frac{100}{d_{p}}\right)^{0.234}
$$

the previous correlation is valid for the following ranges: spherical nanoparticles shapes of 10-150 nm diameter, thermal conductivity of $20-400 \mathrm{~W} / \mathrm{m} . \mathrm{K}$, base fluid having thermal conductivity of $0.1-0.7 \mathrm{~W} / \mathrm{m} . \mathrm{K}$, volume fraction of 0.1 to $3 \%$ and temperature range from 293 to $333 \mathrm{~K}$. 


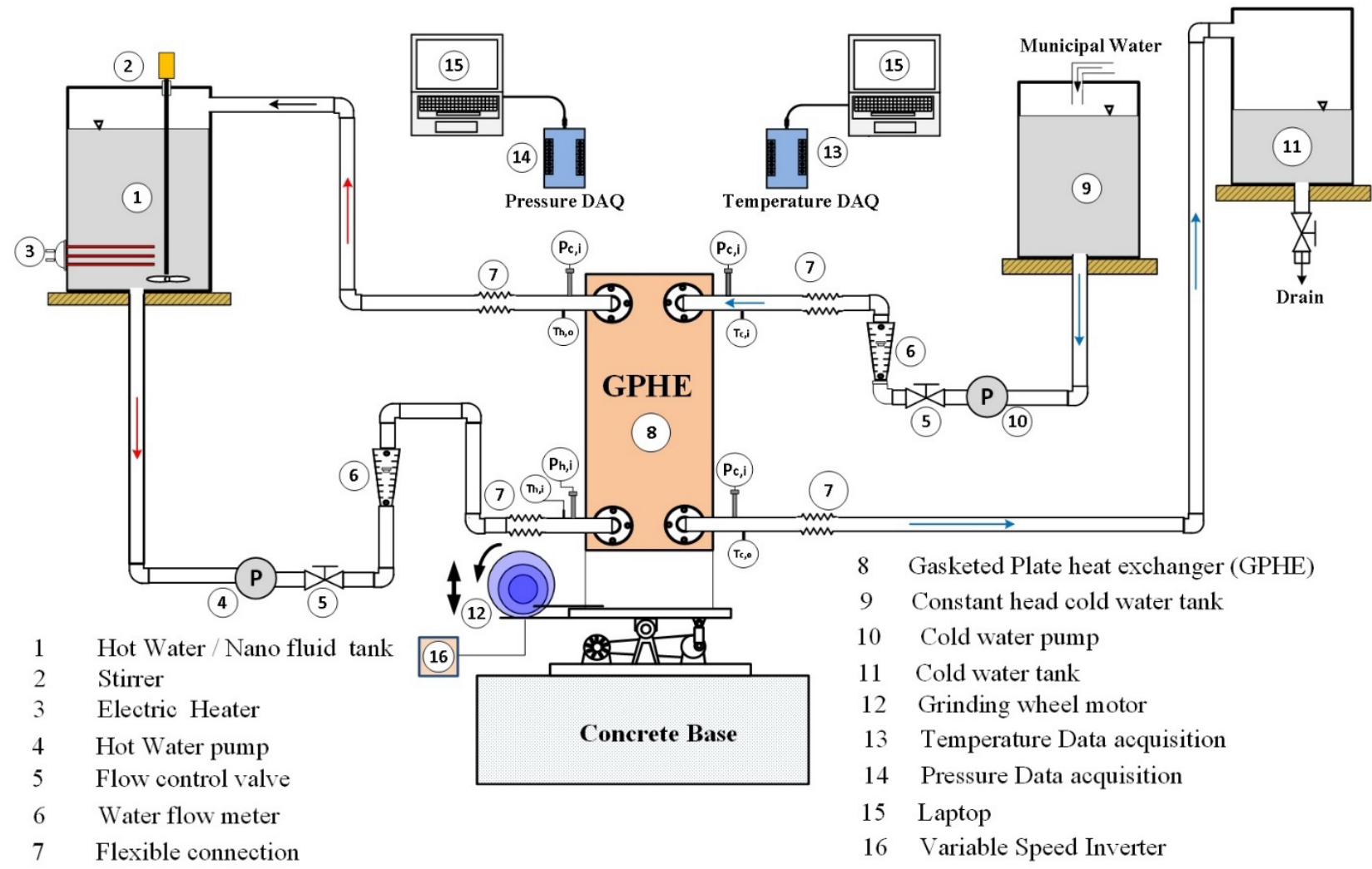

Fig. 2. Schematic diagram of the test rig with instrumentation

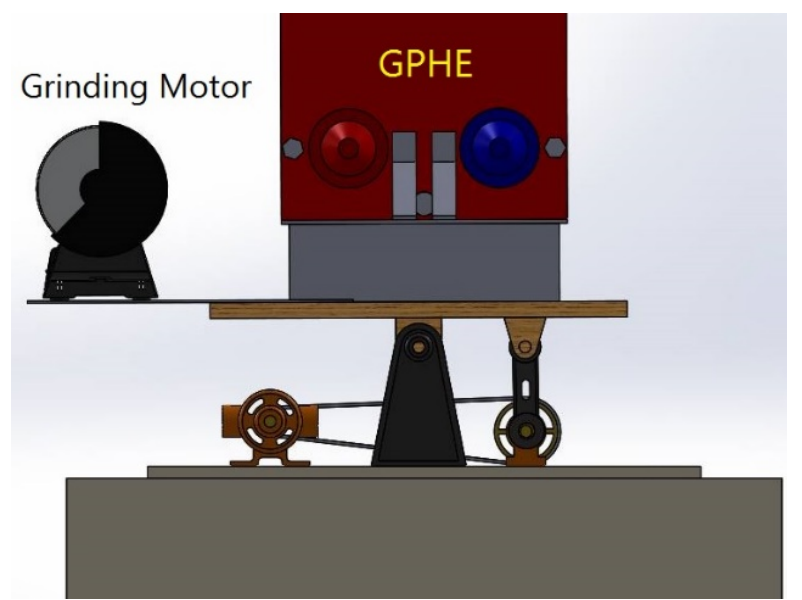

Fig. 3. The location of the grinding motor with respect to the GPHE's platform.

Wang et al.[30] proposed an equation to calculate the viscosity of nanofluid as follows:

$$
\frac{\mu_{e f f}}{\mu_{f}}=1+7.3 \emptyset_{V}+123 \emptyset_{V}^{2}
$$

To obtain nanofluid thermophysical properties as a function with temperature. The base fluid (distilled water) properties as a function of temperature may be obtained as follows, Arani and Amani [31]:

$$
\begin{aligned}
\rho_{b f}=- & 764.475639+19.251515 T \\
& -0.07714568 T^{2} \\
& +1.364893 \times 10^{-4} T^{3} \\
& -9.339158 \times 10^{-8} T^{4}
\end{aligned}
$$

$$
\begin{aligned}
& C_{p . b f}=198531.690492-2894.853934 T \\
&+ 17.2363068 T^{2} \\
&-0.05126994 T^{3} \\
&+ 7.616133 \times 10^{-5} T^{4} \\
&- 4.517821 \times 10^{-8} T^{5} \\
& \ln \left(\frac{\mu_{b f}}{0.001792}\right)=-1.24-6.44\left(\frac{273.15}{T}\right) \\
&+ 7.68\left(\frac{273.15}{T}\right)^{2} \\
& K_{b f}=-1.549404+0.01553952 T \\
&- 3.65967 \times 10^{-5} T^{2} \\
&+ 2.9401 \times 10^{-8} T^{3}
\end{aligned}
$$

All of the previously mentioned above correlations are valid within the temperature range $273.15 \leq \mathrm{T}(\mathrm{K}) \leq 373$.

The dimensionless Reynolds number of hot nanofluid, and cold municipal water can be calculated as follows:

$$
\begin{gathered}
R e_{n f}=\frac{G_{n f} \cdot D_{e}}{\mu_{n f}} \\
R e_{c}=\frac{G_{c} \cdot D_{e}}{\mu_{c}}
\end{gathered}
$$

where the equivalent diameter $\mathrm{De}=2 \mathrm{~b}$. However, the channel mass velocity of hot nanofluid and cold municipal water are defined as follows:

$$
\begin{aligned}
G_{n f} & =\left(\frac{m_{n f}}{N_{\text {c.p.nf }} \cdot b \cdot L_{w}}\right) \\
G_{c} & =\left(\frac{m_{c}}{N_{\text {c.p.c. }} \cdot b \cdot L_{w}}\right)
\end{aligned}
$$


where, the number of channel per pass, $N_{c . p}$ is calculated from

$$
N_{c . p}=\left(\frac{N t-1}{2 . N_{p}}\right)
$$

The heat is transferred from the hot nanofluid to the cold municipal water, are estimated via (15) and (16) using the measured flow rate and temperature.

$$
\begin{gathered}
Q_{n f}=m_{n f} \cdot C p_{n f} \cdot\left(T_{n f . i}-T_{n f . o}\right) \\
Q_{c}=m_{c} \cdot C p_{c} \cdot\left(T_{c o}-T_{c i}\right)
\end{gathered}
$$

where, $\dot{\mathrm{m}}, \mathrm{Cp}$ and $\mathrm{T}$ are the mass flow rate, specific heat and temperature of the inlet (i) and outlet (o) for the hot (nf) and cold (c) respectively. If the difference between the two rates of heat transfer of hot nanofluid and cold municipal water is less than $5 \%$, the experimental data are considered to be reasonable.

The overall heat transfer coefficient (U) for GPHE was evaluated based on the averaged heat transfer rate $\left(Q_{a v}\right)$.

$$
\begin{gathered}
Q_{a v}=\left(Q_{n f}+Q_{c}\right) / 2 \\
U=\frac{Q_{a v}}{A \cdot L M T D}
\end{gathered}
$$

The overall heat transfer coefficient is calculated by using (18) based on the experimental data. Where; the logarithmic mean temperature difference is calculated as follow:

$$
L M T D=\frac{\left(T_{n f . i}-T_{c . o}\right)-\left(T_{n f . o}-T_{c . i}\right)}{\ln \frac{T_{n f . i}-T_{c . o}}{T_{n f . o}-T_{c . i}}}
$$

The heat transfer coefficient of the cold municipal water can be calculated by using the following equation [25].

$$
N u_{c}=0.0423 \cdot R e_{c}^{0.791} \cdot \operatorname{Pr}_{c}^{\frac{1}{3}}\left(\frac{\mu_{c}}{\mu_{w}}\right)^{0.14}
$$

Knowing the thermal conductivity of the plate, kw, and its thickness, $t$, the heat transfer coefficient of the hot nanofluid, $\left(h_{n f}\right)$, has been evaluated using overall heat transfer coefficient and cold water heat transfer coefficient, from the following relationship:

$$
\frac{1}{U}=\frac{1}{h_{n f}}+\frac{t}{k_{w}}+\frac{1}{h_{c}}
$$

Dynamic viscosity at wall conditions, $\left(\mu_{w}\right)$, is determined at the average of hot and cold stream temperatures given as:

$$
T_{\text {wall }}=T_{s}=\left(T_{n f . i}+T_{n f . o}+T_{c i}+T_{c o}\right) / 4
$$

The experimental Nusselt number can be calculated as [32]:

$$
N u=\frac{h \cdot D_{e}}{k_{f}}
$$

Nusselt number as a function of the Reynolds number, Prandtl number and the ratio of the dynamic viscosities at bulk to wall temperature could be calculated by the following equation [32]:

$$
N u=c \cdot \operatorname{Re}^{p} \cdot \operatorname{Pr}^{n} \cdot\left(\frac{\mu}{\mu_{w}}\right)^{d}
$$

To simplify the calculations, the coefficients (n), and (d) can be found from the literature. Therefore, in the present study (n), and (d) are taken as constant as $1 / 3$ and 0.14 , respectively.

The operating parameter known as the Reynolds number oscillation $\left(\mathrm{Re}_{\mathrm{osc}}\right)$, can be calculated as follow:

$$
R e_{o s c}=[(A . \omega \cdot D e) /(\mu / \rho)]
$$

where $A$ is the amplitude of vibration $(\mathrm{mm})$ and $\omega$ is the frequency of oscillation $(\mathrm{Hz})$. The product $\left(\mathrm{A}^{*} \omega\right)$ is called vibration intensity. The geometrical parameter known as the dimensionless oscillation amplitude $(\mathrm{A} / \mathrm{De})$, is the ratio of the vibration amplitude to the PHE channel equivalent diameter.

The measured pressure drops on hot nanofluid and cold municipal water sides are calculated from experimental observations and theoretical formula as given below as:

$$
\Delta P_{n f}=\left(P_{n f . i}-P_{n f . o}\right) \& \Delta P_{c}=\left(P_{c . i}-P_{c . o}\right)
$$

The total measured pressure drop on one fluid side in a PHE has been evaluated by the following relationship [33]:

$$
\begin{aligned}
\Delta P=\left[\frac{1 \cdot 5 \cdot G_{p}^{2} \cdot N_{p}}{2 \cdot g_{c} \cdot \rho_{i}}\right] & +\left[\frac{4 \cdot f \cdot L \cdot G^{2}}{2 \cdot g_{c} \cdot D_{e}}\right]\left[\frac{1}{\rho}\right]_{m} \\
& +\left[\frac{1}{\rho_{o}}-\frac{1}{\rho_{i}}\right]\left[\frac{G^{2}}{g_{c}}\right] \pm\left[\frac{\rho_{m} \cdot g \cdot L}{g_{c}}\right]
\end{aligned}
$$

From the measured total pressure drop across the PHE for both the hot nanofluid and cold water streams, the core friction pressure drop can be obtained as follow [33].

$$
\begin{gathered}
\Delta P_{\text {core }}=\Delta P_{\text {measured }}-\Delta P_{\text {port }} \pm \Delta P_{\text {elevation }} \\
-\Delta P_{\text {momentum }}
\end{gathered}
$$

Therefore, by using the measured pressure drop during experiments, and the tested PHE geometrical specifications, the friction factor of a corrugated PHE can be found as follow:

$$
f=\left[\frac{\Delta P_{\text {core }} \cdot \rho_{m} \cdot g_{c} \cdot D_{e}}{2 \cdot L \cdot G^{2} \cdot}\right]
$$

By using a curve fitting (a nonlinear regression) an empirical correlation for friction factor can be obtained.

\section{Experimental uncertainty analysis}

Let $w_{\mathrm{R}}$ be the uncertainty in the results and $w_{1}, w_{2}, w_{3}, \ldots, w_{\mathrm{n}}$ be the uncertainties in the independent variables. If the uncertainties in the independent variables are all given with the same odds, then the uncertainty in the results having these odds is given by the following equation [34]:

$$
\begin{aligned}
w_{R}=\left[\left(\frac{\partial R}{\partial X_{1}} w_{1}\right)^{2}\right. & +\left(\frac{\partial R}{\partial X_{2}} w_{2}\right)^{2} \\
& \left.+\cdots\left(\frac{\partial R}{\partial X_{n}} w_{n}\right)^{2}\right]^{0.5}
\end{aligned}
$$

The experimental error analysis for all used working parameters was checked according to Holman's method [34]. The uncertainty calculations showed maximum value of \pm 3.22 , $\pm 5.52, \pm 5.56, \pm 3.85$ and \pm 5.0 in results for Reynolds number, friction factor, heat transfer rate, overall heat transfer coefficient, and net augmentation Nusselt number, respectively, which are within the acceptable range.

\section{RESUlts AND DisCUSSION}

The present study cover four GPHE models. The first model is conventional or base GPHE (without nano-particles and vibrations). The 2nd model is GPHE with nano-particles only. The 3rd model is Bassiouny et al. [25] model (GPHE with vibration only). Finally, the 4th model is GPHE with nanoparticles and vibrations. All results will be relative to the $1 \mathrm{st}$ model. 
A. .Heat transfer augmentation for GPHE with nanofluid only

Fig. 4 indicates the relationship between the nanofluid convection heat transfer coefficient enhancement ratio $h_{n} / h_{o}$ and the flow Reynolds number for stationary GPHE $\left(\mathrm{Re}_{\mathrm{osc}}=\right.$ 0.0 ) at four different nanofluid volume fractions. The results demonstrate that the nanofluid convection heat transfer coefficient enhancement ratio increases with increasing the nanofluid volume fraction. The best enhancements in nanofluid convection heat transfer coefficient enhancement ratio are $4.9 \%, 7.9 \%, 10.6 \%$, and $14 \%$ that accomplished at nanofluid volume fractions of $\left(\emptyset_{V}=0.125 \%, 0.25 \%, 0.378 \%\right.$, and $0.51 \%$ ) respectively. The reason for these enhancements may be happened because of nanoparticles Brownian motion, thermal diffusion, and thermophoresis. The Brownian motion of $\mathrm{Al}_{2} \mathrm{O}_{3}$ nanoparticles led to reduce the thickness of thermal boundary layer $\left(\delta_{t}\right)$ and at the same time increases the nanofluid thermal conductivity $\left(k_{n f}\right)$ [35]. The reduction in thermal boundary layer thickness $\left(\delta_{t}\right)$ and the enhancing in nanofluid thermal conductivity $\left(k_{n f}\right)$, both of them increasing the nanofluid augmentation coefficient $h_{n} / h_{o}$.

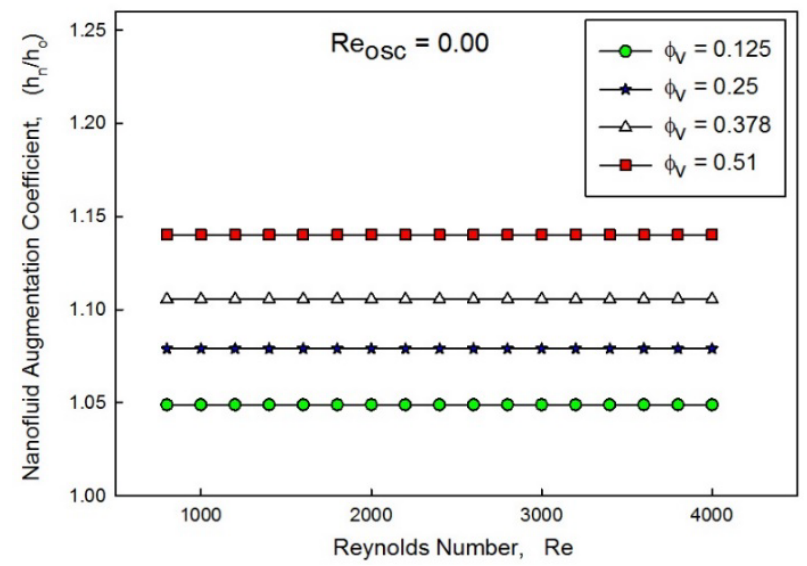

Fig. 4. Effect of flow Reynolds number on the nanofluid convection heat transfer coefficient enhancement ratio at different nanofluid volume fraction for stationary GPHE.

\section{B. Net heat transfer augmentation for GPHE with nanofluids and vibration.}

Fig. 5 shows the variation of net convection heat transfer coefficient enhancement ratio $h_{n+v} / h_{o}$ ) with flow Reynolds number for oscillation Reynolds number $\left(\mathrm{Re}_{\mathrm{osc}}=211.34\right)$ at five different nanofluid volume fractions. It could be seen from this Figure that the value of net convection heat transfer coefficient enhancement ratio increases as the flow Reynolds number and nanofluid volume fraction increases. The maximum increases are $44 \%, 51 \%, 55 \%, 59 \%$, and $64 \%$ that take place at $\left(\emptyset_{V}=0.0 \%, 0.125 \%, 0.25 \%, 0.378 \%\right.$, and $\left.0.51 \%\right)$ respectively. It could be noted that the maximum increases in this model is grather than that for the 2nd model (GPHE with nano-particles only) and the 3rd model (Bassiouny et al. [25] model, GPHE with vibration only) by about $50 \%$ and $21 \%$ respectively. This is because the enhanced turbulence intensity due to the collisions among nano-particles and with plate surface and water that cause tearing of the boundary layer. Also, the augmentation of thermal diffusion and thermophoresis are consider an important reason for heat transfer enhancement. Furthermore, the vibrations decrease the sedimentation of nanoparticles, that improve the nanofluid heat transfer performance.
Fig. 6 shows the relation between of net convection heat transfer coefficient enhancement ratio and flow Reynolds number at nanofluid volume fraction of $\left(\emptyset_{V}=0.51 \%\right)$ and different oscillation Reynolds number of $\left(\operatorname{Re}_{\mathrm{osc}}=0.0,16.29\right.$, $71.92,110.56,159.50$ and 211.34$)$. As can be seen, for all oscillation Reynolds number, as the flow Reynolds number increases, the net convection heat transfer coefficient enhancement ratio increases. The net convection heat transfer coefficient enhancement ratio changes with flow Reynolds number is insignificant and equal to $14 \%$. The most enhancements in the net convection heat transfer coefficient enhancement ratio are $14 \%, 38 \%, 41 \%, 49.4 \%, 55 \%$, and $64 \%)$ which obtained at oscillation Reynolds numbers of $\left(\mathrm{Re}_{\mathrm{osc}}\right.$ $=0.0,71.92,16.29,110.56,159.47$ and 211.34) respectively. This is because, the vibrated GPHE increase flow turbulence close to the boundary layer and hence decrease its thickness. This lead up to a good enhancement in heat transfer as indicated by Benhamou [36]. Also, the secondary flow that occurs in the transversal direction that result from the vertical vibration force. This causes convective currents across the GPHE section, and induce a strong momentum transfer, that augment the heat transfer performance. Finally, a result of using nanofluid the heat transfer coefficients was significantly enhanced [37].

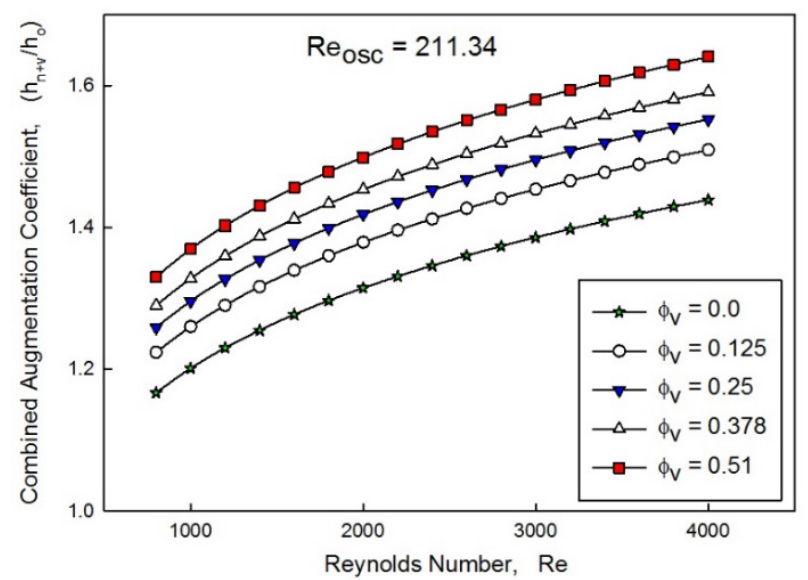

Fig. 5. Effect of flow Reynolds number on the net convection heat transfer coefficient enhancement ratio at five different nanofluid volume fractions, for GPHE oscillation Reynolds number $=211.34$.

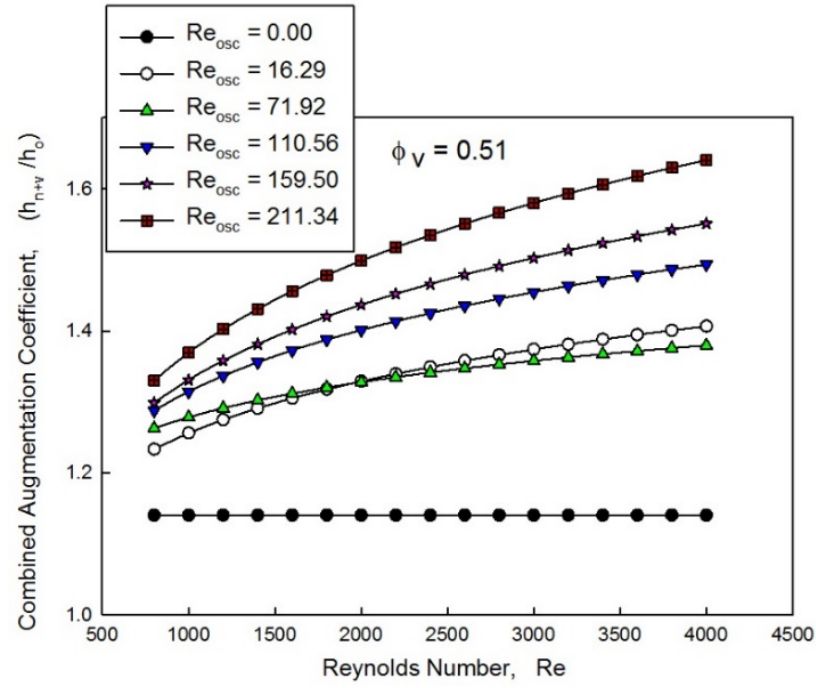

Fig. 6. Effect of flow Reynolds number on the vibration augmentation coefficient at different GPHE oscillation Reynolds number, for nanofluid volume fraction of $0.51 \%$. 
C. Net augmentation heat transfer Nusselt number correlation steps and methodology.

The measured data obtained from the experimental test were used to calculate Reynolds number for both hot and cold fluid sides. LMTD and heat balance equation are used to deduce the overall heat transfer coefficient from eq. (18). Then, the heat transfer coefficient towards the hot nanofluid side can be calculated by using eq. (21). To figure out the influence of nanofluid volume fraction $\left(\varphi_{v}\right)$ on heat transfer Nusselt number correlation eq. (24), Fig. 7 illustrates the trend of the augmented heat transfer coefficient against the hot nanofluid Reynolds number.

can be written on an alternated form as:

$$
\begin{gathered}
h=\alpha \cdot C \cdot \operatorname{Re}^{P} \\
\alpha=\operatorname{Pr}^{1 / 3}\left(\frac{\mu}{\mu_{s}}\right)^{0.14} \frac{K}{D_{e}}
\end{gathered}
$$

Then, the logarithmic scale of both sides of last equation can be rewritten in the form:

$$
\ln \left(\frac{h}{\alpha}\right)=\ln (C)+p \cdot \ln (R e)
$$

After that, we determine the ratio between the case of combined (vibration + nanofluid) augmented heat transfer and vibration augmented heat transfer as follows;

$$
\ln \left(\frac{h_{n+v}}{h_{v}} \cdot \frac{\alpha_{v}}{\alpha_{n+v}}\right)=\ln \left(\frac{C_{n+v}}{C_{v}}\right)+\left(P_{n+v}-P_{v}\right) \cdot \ln (R e)
$$

where the left hand side represents the Y-axis of Fig. 7, and the last term $\ln (R e)$ represents its $\mathrm{X}$-axis. Furthermore, the constant term indicates the logarithmic scale of ratio, $\left(C_{n+v} / C_{v}\right)$ while the curve slope shows the difference between the two indexes $\left(P_{n+v}-P_{v}\right)$. The linear regression between the two variables $(\mathrm{X} \& \mathrm{Y})$, eventually shows that nanofluid augmented coefficient is a strong function in nanofluid volume fraction $\left(\varphi_{v}\right)$, whereas the effect of increasing Reynolds number can be insignificant. As a result, we can deduce that nanofluid augmentation coefficient is a fixed relation in oscillation Reynolds number (independent of Reynolds number index).

In Fig. 8 the ratio $\left(C_{n+v} / C_{v}\right)$ was plotted against the tested range of nanofluid volume fraction $\left(\varphi_{v}\right)$. On the other side, the solid line indicates the linear regression of $\left(1+\varphi_{v}\right)$ and $\left(C_{n+v} / C_{v}\right)$ in logarithmic scale. The resultant relation is well fitted with a slope of 0.165 , then we can rewritte the equation in the form;

$$
C_{n+v} / C_{v}=\left(1+\varphi_{v}\right)^{0.165}
$$

Finally, the proposed net augmentation Nusselt number correlation as a function of Reynolds number (Re), oscillation Reynolds number $\left(\mathrm{Re}_{\mathrm{osc}}\right)$ and dimensionless vibration amplitudeis $\left(\mathrm{A} / \mathrm{D}_{\mathrm{e}}\right)$ and nanofluid volume fraction $\left(\emptyset_{V}\right)$ can be suggested in the following form:

$$
N u_{(n+v)}=\left(1+\varphi_{v}\right)^{0.165} \cdot C_{v} \cdot R e^{P_{v}}
$$

Where the constant $C_{v}$ and index $P_{v}$ in the vibration enhancement case have two different trends, first of them belongs to all oscillation Reynolds number that occur before resonance condition till the largest value of oscillation Reynolds number $\left(\mathrm{Re}_{\mathrm{osc}}=211.34\right)$ that take place just after the resonance condition. Below grinding motor speed of $1800 \mathrm{rpm}$ (i.e. at $\mathrm{Re}_{\mathrm{osc}}=211.34$ ), both vibration frequency and vibration amplitude are increased, that cause increasing of oscillation Reynolds number as depicted in Fig. 9. The second trend belongs to all oscillation Reynolds number $\left(\mathrm{Re}_{\mathrm{osc}}\right)$ with 2500 and $2800 \mathrm{rpm}$ that take place after and far from the resonance condition, In this period, as grinding motor speed increases, Reynolds number oscillation decreases. This is because vibration amplitude is decreased as shown in Fig. 9.

In the first trend, the constant $C_{v}$ and index $P_{v}$ are changed according to the following correlations:

$$
\begin{gathered}
C_{v}=0.0423-2.1 * 10^{-4} \cdot R e_{\text {osc }}^{0.531} \cdot\left(\frac{A}{D_{e}}\right)^{-0.605} \\
P_{v}=0.791+1.883 * 10^{-4} \cdot \operatorname{Re}_{\text {osc }}{ }^{0.7533} \\
\cdot\left(\frac{A}{D_{e}}\right)^{-0.846}
\end{gathered}
$$

However, In the second trend, the constant $C_{v}$ and index $P_{v}$ are changed according to the others different following correlations:

$$
\begin{gathered}
C_{v}=0.0423-1.4344 * 10^{-18} \cdot \operatorname{Re}_{\text {osc }}^{5.21} \\
\cdot\left(\frac{A}{D_{e}}\right)^{-3.18} \\
P_{v}=0.791+1.9874 * 10^{-17} \cdot \operatorname{Re}_{o s c}^{5.096} \\
\cdot\left(\frac{A}{D_{e}}\right)^{-3.085}
\end{gathered}
$$

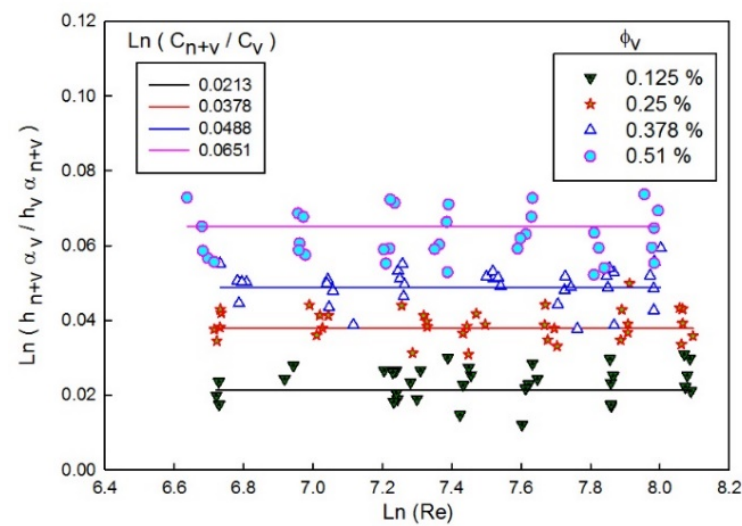

Fig. 7. The linear regression between the two variables (X \& Y), where $Y$ axis (ratio of combined to vibrated augmented coefficient) against $X$-axis (hot nanofluid Reynolds number), both axis in logarithmic scale.

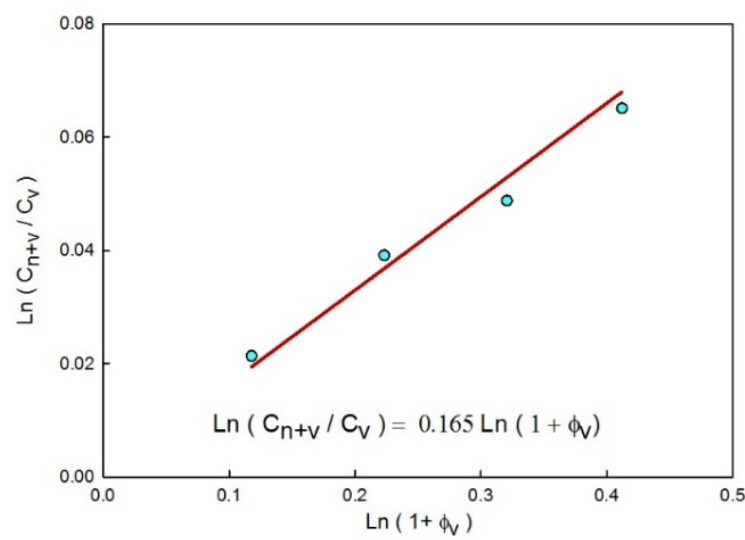

Fig. 8. The linear regression between the ratio $\left(\frac{C_{n+v}}{C_{v}}\right)$ against the tested range of nanofluid volume fraction $\left(\varphi_{v}\right)$. 


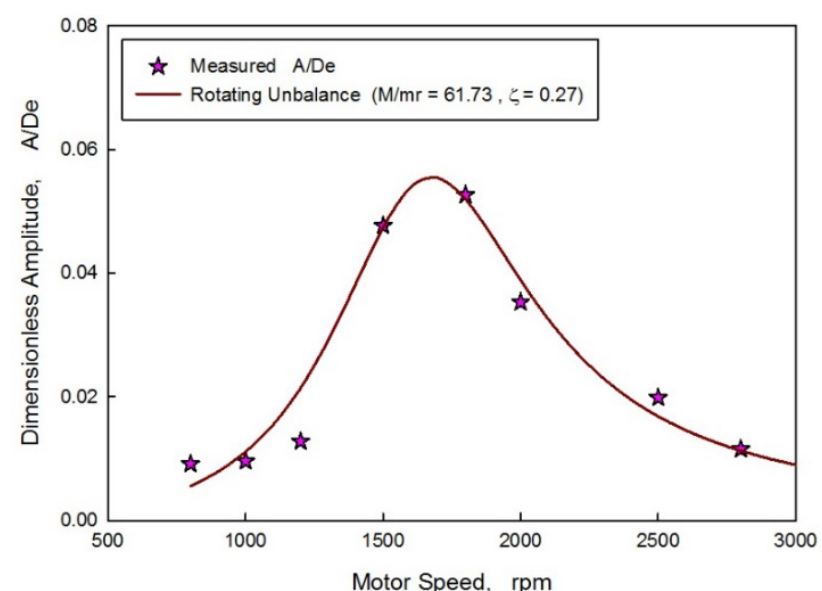

Fig. 9 Change of vibration amplitude with the grinding motor speed measured at three points on the PHE.

\section{Pressure drop for GPHE with nanofluid only.}

The relation between the measured pressure drop $(\Delta \mathrm{P})$ across the GPHE and the Reynolds number for hot nanofluid and purified distilled water is illustrated in Fig. 10. It could be seen from this Fig. that the pressure drop increases as nanofluid Reynolds number increases. At a certain nanofluid Reynolds number, pressure drop was the least for purified distilled water and it slightly increased with the nanofluid volume fraction $\left(\emptyset_{V}\right)$ increases. The pressure drop through the GPHE is a function of nanofluid Reynolds number, density and viscosity as well as the GPHE geometrical parameters.

Viscosity of nanofluid increases as the nanofluid volume fraction increases while the nanofluid density practically remains constant. The experiments were executed at constant Reynolds number as depicted from Fig. 10. To achieving the condition of constant Reynolds number after the nanofluid volume fractions are increasing, more volumetric flow rate is needed to recover the viscosity increases, the cause behind increase both of the pressure losses and the needed pumping power. The most increase in pressure loss has obtained relative to the purified distilled water case are $\left(\Delta P_{n}=11 \%, 16.4 \%\right.$, $20.6 \%$, and $23.5 \%)$ which take place at $\left(\emptyset_{V}=0.125 \%, 0.25 \%\right.$, $0.378 \%$, and $0.51 \%$ ) respectively, at Reynolds number approximatly equal of 3000 .

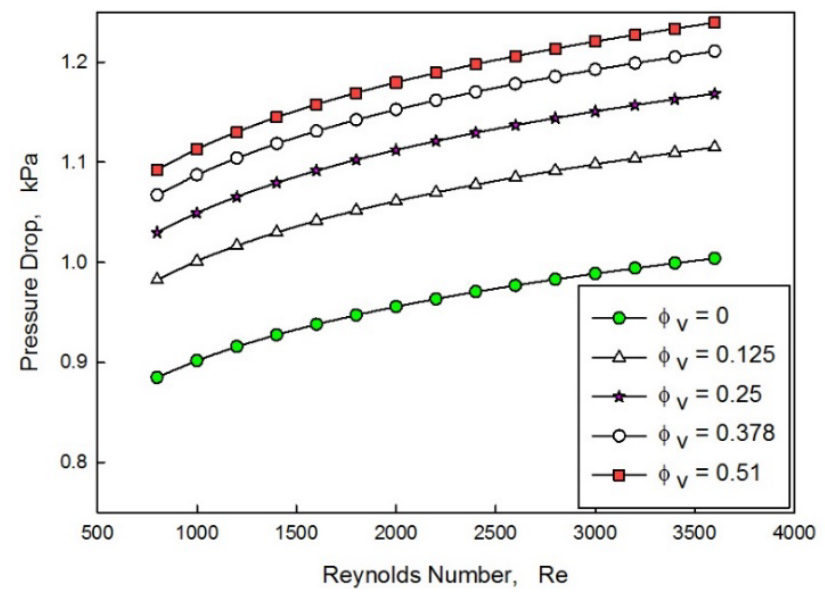

Fig. 10 The GPHE measured pressure drop versus the Reynolds number for nanofluid and purified water, with different nanofluid volume fractions.

\section{E. Stationary GPHE friction factor with nanofluid}

In Fig. 11 indicates the variation of the GPHE friction factor $(f)$ with the hot nanofluid Reynolds number ( $R e)$ for both nanofluid and purified water. The proposed correlation for predicting the GPHE friction factor $(f)$ is found to be:

$$
f=A_{2} \cdot R e^{-1.916}
$$

where $A_{2}=\left(5.14+4.25 \emptyset_{V}-4.11 \emptyset_{V}^{2}\right) \times 10^{7}$.

This empirical correlation for friction factor can be obtained by using a curve fitting (a nonlinear regression) as depicted in Fig. 12.

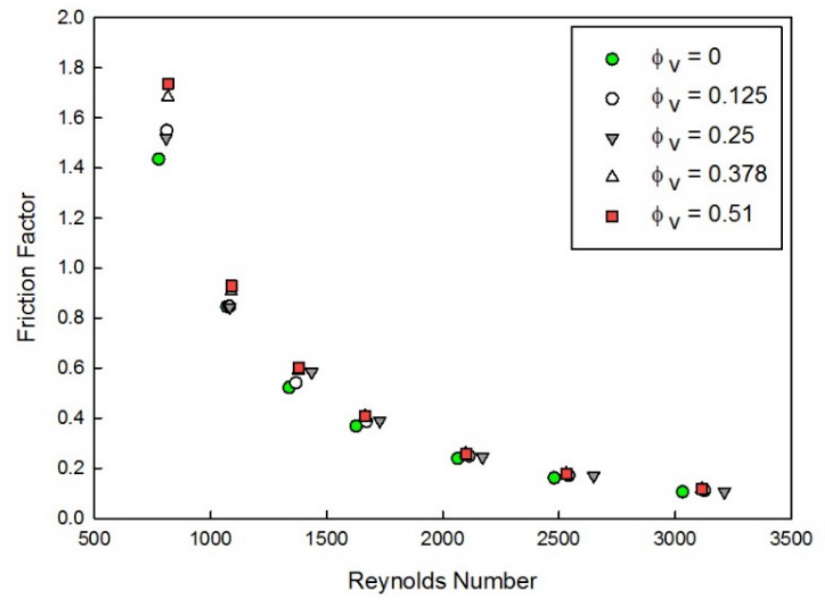

Fig. 11 The GPHE friction factor against the Reynolds number for nanofluid and purified water, with different nanofluid volume fractions.

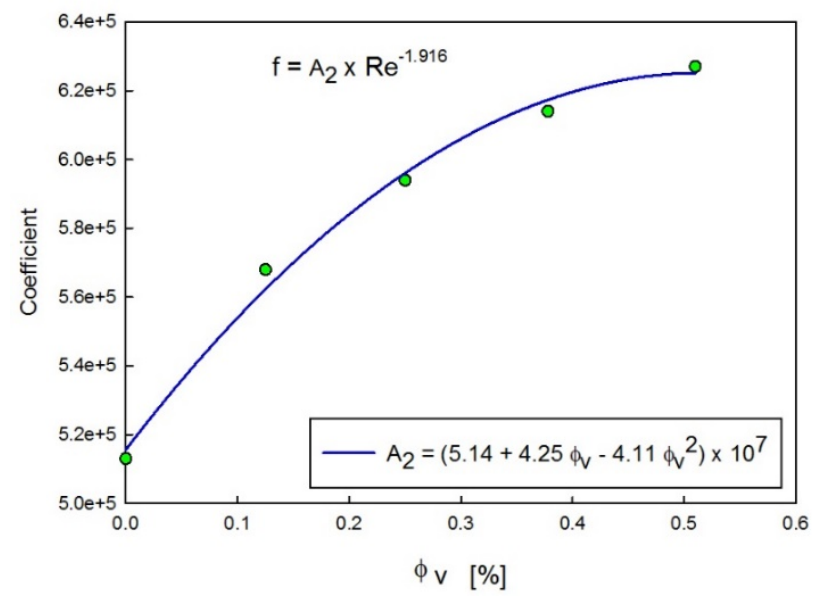

Fig. 12 Variation of friction factor correlation coefficent with nanofluid volume fractions.

\section{CONCLUSIONS}

In the present study, the effect of applying a low amplitude mechanical vibrations on a gasketed plate heat exchanger (GPHE), with $\gamma \mathrm{Al}_{2} \mathrm{O}_{3}$-water nanofluid as a combined augmentation technique for heat transfer enhancement was experimently studied. Applying the surface vibrations augments the heat transfer coefficients, while reduces the sedimentation of nanoparticles. According to the analysis of the results obtained from experimental study, the main conclusions from the present study can be summarized as follow:

1- Using $\gamma \mathrm{Al}_{2} \mathrm{O}_{3}$-water nanofluid as a working fluid in the hot loop enhances heat transfer performance of the GPHE with a relatively increase in pressure drop. 
2- The nanofluid convection heat transfer enhancement ratio increases with increasing nanofluid volume fraction and the maximum augmentation is $14 \%$ at nanofluid volume fraction of $\left(\emptyset_{V}=0.51 \%\right)$.

3- The influence of combined augmentation that includes the application of nanofluid and vibration of heat transfer surface operating simultaneously on the heat transfer performance enhancement is greater than that obtained by using separable techniques working individually by about 16 to $45 \%$. The net convection heat transfer enhancement ratio is observed to be a maximum of $64 \%$, when applied net augmentation of $\mathrm{Al}_{2} \mathrm{O}_{3}$-water nanofluid with highest volume fraction of $\left(\varnothing_{V}=0.51 \%\right)$, and mechanical vibration with Reynolds number oscillation $\left(\mathrm{Re}_{\mathrm{osc}}=\right.$ 211.34).

4- Below grinding motor speed of $1800 \mathrm{rpm}$ (i.e. at $\mathrm{Re}_{\mathrm{osc}}=$ 211.34), both vibration frequency and vibration amplitude are directly proportional to GPHE heat transfer enhancement ratio.

However, for higher grinding motor speed, vibration frequency is increased and both vibration amplitude and GPHE heat transfer enhancement ratio is decreased.

5- New net augmentation Nusselt number correlation as a function of Reynolds number, oscillation Reynolds number and dimensionless vibration amplitude is and nanofluid volume fraction suggested with a relative error of $5 \%$.

\section{ACKNOWLEDGEMENT}

The authors are grateful to Science and Technology Development Fund (STDF), Egypt, grant ID 4872, for supporting the equipment used for preparing nanofluids in this study. Also, the authors grateful to Mechanics Research Institute and Electricity - National Center for Water Research, Egypt for borrowing the device (MVP-2C One Prod) for vibration measurements during doing the experimental work.

\section{NOMENClAtURE}

$\begin{array}{ll}A_{1} & \text { Effective heat transfer area, }\left[\mathrm{m}^{2}\right] \\ A_{2} & \text { Friction factor correlation coefficient. } \\ \mathrm{A}, \text { or } \mathrm{S} & \text { Vibration amplitude, [mm]. } \\ \mathrm{b} & \text { Plate corrugation depth or mean channel spacing, [m]. } \\ \mathrm{C}_{\mathrm{p}} & \text { Specific heat at constant pressure, [J/kg. K]. } \\ \mathrm{C} & \text { Mass capacitance rate, [W/K]. } \\ \mathrm{C}_{\mathrm{R}} & \text { Heat capacity rate ratio, [dimensionless]. } \\ \mathrm{D} & \text { Diameter, [m]. } \\ \mathrm{D}_{\mathrm{e}} & \text { Equivalent diameter of flow passages, [m]. } \\ d_{p} & \text { Port diameter, [m]. } \\ \mathrm{G} & \left.\text { Channel mass velocity, [kg/m }{ }^{2} . \mathrm{s}\right] . \\ \mathrm{h} & \text { Convection heat transfer coefficient, [W/m². K]. } \\ \mathrm{k} & \text { Effective thermal conductivity, [W/m. K]. } \\ \mathrm{L}_{\mathrm{p}} & \text { Effective plate length, }(\mathrm{m}) . \\ \mathrm{L}_{\mathrm{w}} & \text { Effective plate width, [m]. } \\ \dot{\mathrm{m}} & \text { Mass flow rate, [kg/s]. } \\ N_{c p} & \text { Number of channels per pass. } \\ N_{e f f} & \text { Number of all thermally effective plates } \\ N_{p} & \text { Number of flow passes on the given fluid side. } \\ N_{t} & \text { Total number of GPHE plates. } \\ N u & \text { Nusselt number, [dimensionless]. } \\ N u_{n+v} & \text { Combined Nusselt number, [dimensionless]. } \\ \mathrm{P} & \text { Pressure, [Pa or kPa]. } \\ \mathrm{Pc} & \text { Plate corrugation pitch, [m]. } \\ \mathrm{Pr} & \text { Prandtl number, [dimensionless]. } \\ & \end{array}$

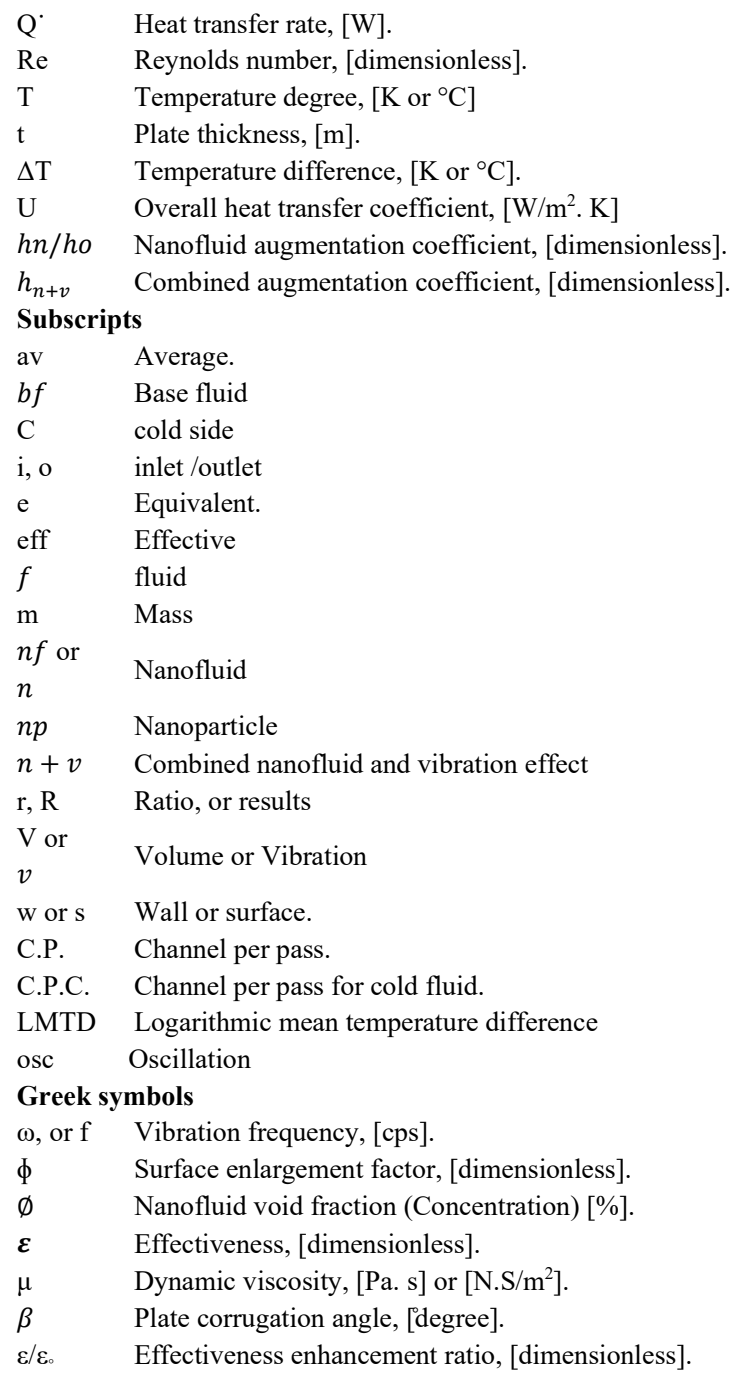

\section{REFERENCES}

[1] S. Chol and J. Estman, "Enhancing thermal conductivity of fluids with nanoparticles," ASME-Publications-Fed, vol. 231, pp. 99-106, 1995.

[2] L. Godson, B. Raja, D. M. Lal, and S. Wongwises, "Enhancement of heat transfer using nanofluids - an overview," Renewable and sustainable energy reviews, vol. 14, pp. 629-641, 2010.

[3] W. Daungthongsuk and S. Wongwises, "A critical review of convective heat transfer of nanofluids," Renewable and sustainable energy reviews, vol. 11, pp. 797-817, 2007.

[4] X.-Q. Wang and A. S. Mujumdar, "Heat transfer characteristics of nanofluids: a review," International journal of thermal sciences, vol. 46, pp. 1-19, 2007

[5] S. Kakac and A. Pramuanjaroenkij, "Review of convective heat transfer enhancement with nanofluids," International Journal of Heat and Mass Transfer, vol. 52, pp. 3187-3196, 2009.

[6] J. Sarkar, "A critical review on convective heat transfer correlations of nanofluids," Renewable and Sustainable Energy Reviews, vol. 15, pp. 3271-3277, 2011.

[7] G. Huminic and A. Huminic, "Application of nanofluids in heat exchangers: a review," Renewable and Sustainable Energy Reviews, vol. 16 , pp. 5625-5638, 2012.

[8] M. Pantzali, A. Kanaris, K. Antoniadis, A. Mouza, and S. Paras, "Effect of nanofluids on the performance of a miniature plate heat exchanger with modulated surface," International Journal of Heat and Fluid Flow, vol. 30, pp. 691-699, 2009

[9] M. Pantzali, A. Mouza, and S. Paras, "Investigating the efficacy of nanofluids as coolants in plate heat exchangers (PHE)," Chemical Engineering Science, vol. 64, pp. 3290-3300, 2009

[10] T. Maré, S. Halelfadl, O. Sow, P. Estellé, S. Duret, and F. Bazantay, "Comparison of the thermal performances of two nanofluids at low temperature in a plate heat exchanger," Experimental Thermal and Fluid Science, vol. 35, pp. 1535-1543, 2011.

[11] A. Zamzamian, S. N. Oskouie, A. Doosthoseini, A. Joneidi, and M. Pazouki, "Experimental investigation of forced convective heat transfer 
coefficient in nanofluids of $\mathrm{Al} 2 \mathrm{O} 3 / \mathrm{EG}$ and $\mathrm{CuO} / \mathrm{EG}$ in a double pipe and plate heat exchangers under turbulent flow," Experimental Thermal and Fluid Science, vol. 35, pp. 495-502, 2011.

[12] Y. Kwon, D. Kim, C. Li, J. Lee, D. Hong, J. Lee, S. Lee, Y. Cho, and S. Kim, "Heat transfer and pressure drop characteristics of nanofluids in a plate heat exchanger," Journal of nanoscience and nanotechnology, vol. 11, pp. 5769-5774, 2011.

[13] S. D. Pandey and V. Nema, "Experimental analysis of heat transfer and friction factor of nanofluid as a coolant in a corrugated plate heat exchanger," Experimental Thermal and Fluid Science, vol. 38, pp. 248 256, 2012.

[14] A. K. Tiwari, P. Ghosh, and J. Sarkar, "Particle concentration levels of various nanofluids in plate heat exchanger for best performance," International Journal of heat and mass transfer, vol. 89, pp. 1110-1118, 2015.

[15] D. R. Ray, D. K. Das, and R. S. Vajjha, "Experimental and numerical investigations of nanofluids performance in a compact minichannel plate heat exchanger," International Journal of Heat and Mass Transfer, vol 71, pp. 732-746, 2014

[16] V. Kumar, A. K. Tiwari, and S. K. Ghosh, "Application of nanofluids in plate heat exchanger: a review," Energy conversion and management, vol. 105, pp. 1017-1036, 2015.

[17] A. Kabeel, T. A. El Maaty, and Y. El Samadony, "The effect of using nano-particles on corrugated plate heat exchanger performance," Applied Thermal Engineering, vol. 52, pp. 221-229, 2013.

[18] A. M. Abed, M. Alghoul, K. Sopian, H. Mohammed, and A. N. AlShamani, "Design characteristics of corrugated trapezoidal plate heat exchangers using nanofluids," Chemical Engineering and Processing: Process Intensification, vol. 87, pp. 88-103, 2015.

[19] S. Kakaç, A. E. Bergles, F. Mayinger, and H. Yüncü, Heat transfer enhancement of heat exchangers vol. 355: Springer Science \& Business Media, 2013.

[20] K. K. Prasad and V. Ramanathan, "Heat transfer by free convection from a longitudinally vibrating vertical plate," International Journal of Heat and Mass Transfer, vol. 15, pp. 1213-1223, 1972

[21] E. Eid and M. Gomaa, "Influence of vibration in enhancement of heat transfer rates from thin plannar fins," Heat and mass transfer, vol. 45, pp. 713-726, 2009.

[22] A. R. Sarhan, "Vertical forced vibration effect on natural convective performance of longitudinal fin heat sinks," Tikrit Journal of Engineering Sciences, vol. 20, pp. 60-69, 2013.

[23] A. Klaczak, "Report from experiments on heat transfer by forced vibrations of exchangersErgebnisse von Wärmeübergangsversuchen an einem Wärmetauscher mit Schwingungsanregung," Heat and Mass transfer, vol. 32, pp. 477-480, 1997.
[24] P. Chatter and K. Sandeep, "Effect of vibration on heat transfer enhancement in a rectangular channel heat exchanger," IOSR J Mech Civil Eng, pp. 51-57, 2013.

[25] M. K. Bassiouny, A. E. Kabeel, A. Khalil, Y. A. F. El Samadony, and A M. Khaira, "Heat Transfer Performance of a Gasketed Plate Heat Exchanger Subjected to Mechanical Vibration," Tanta Eng. Journal, vol. 3, Feb. 2019. "in press"

[26] I. C. Bang and S. H. Chang, "Boiling heat transfer performance and phenomena of A12O3-water nano-fluids from a plain surface in a pool," International Journal of Heat and Mass Transfer, vol. 48, pp. 2407-2419, 2005.

[27] B. C. Pak and Y. I. Cho, "Hydrodynamic and heat transfer study of dispersed fluids with submicron metallic oxide particles," Experimental Heat Transfer an International Journal, vol. 11, pp. 151-170, 1998.

[28] Y. Xuan and W. Roetzel, "Conceptions for heat transfer correlation of nanofluids," International Journal of heat and Mass transfer, vol. 43, pp. 3701-3707, 2000.

[29] H. E. Patel, T. Sundararajan, S. K. Das, An experimental investigation into the thermal conductivity enhancement in oxide and metallic nanofluids, J. Nanoparticle Res. 12 (3) (2010) 1015-1031.

[30] X. Wang, X. Xu, S. U. S. Choi, Thermal conductivity of nanoparticlefluid mixture, J. Thermophys. Heat Transfer 13(4) (1999) 474-480.

[31] A. A. Arani and J. Amani, "Experimental investigation of diameter effect on heat transfer performance and pressure drop of $\mathrm{TiO} 2-$ water nanofluid," Experimental Thermal and Fluid Science, vol. 44, pp. 520 $533,2013$.

[32] A. Muley, "Heat transfer and pressure drop in plate heat exchangers," 1998.

[33] R. K. Shah and D. P. Sekulic, Fundamentals of heat exchanger design: John Wiley \& Sons, 2003.

[34] J. P. Holman, Experimental methods for engineers, fifth ed.: McGrawHill New York, 1989.

[35] Y. He, Y. Jin, H. Chen, Y. Ding, D. Cang, and H. Lu, "Heat transfer and flow behaviour of aqueous suspensions of $\mathrm{TiO} 2$ nanoparticles (nanofluids) flowing upward through a vertical pipe," International journal of heat and mass transfer, vol. 50, pp. 2272-2281, 2007.

[36] B. Benhamou, N. Galanis, and A. Laneville, "Transient effects of orthogonal pipe oscillations on laminar developing incompressible flow," International journal for numerical methods in fluids, vol. 34, pp. 561-584, 2000.

[37] A. Sergis and Y. Hardalupas, "Anomalous heat transfer modes of nanofluids: a review based on statistical analysis," Nanoscale research letters, vol. 6, p. 391, 2011 